\title{
Discrepancies in Dose-volume Histograms Generated from Different Treatment Planning Systems
}

\author{
Jung-in Kim ${ }^{1,2,3}$, Ji Hye Han ${ }^{4}$, Chang Heon Choi ${ }^{1,2,3}$, Hyun Joon $\mathrm{An}^{1,2,3}$, Hong-Gyun $\mathrm{Wu}^{1,2,3,5}$, Jong Min Park ${ }^{1,2,3,6, *}$ \\ ${ }^{1}$ Department of Radiation Oncology, Seoul National University Hospital, Seoul, Korea; ${ }^{2}$ Institute of Radiation Medicine, Seoul National University Medical \\ Research Center, Seoul, Korea; ${ }^{3}$ Biomedical Research Institute, Seoul National University College of Medicine, Seoul, Korea; ${ }^{4}$ Department of Physics, Ewha \\ Womans University, Seoul, Korea; ${ }^{5}$ Department of Radiation Oncology, Seoul National University College of Medicine, Seoul, Korea; ${ }^{6}$ Robotics Research \\ Laboratory for Extreme Environments, Advanced Institutes of Convergence Technology, Suwon, Korea
}

\section{Original Research}

Received April 2, 2018

Revision May 14, 2018

Accepted May 25, 2018

Corresponding author: Jong Min Park

Department of Radiation Oncology, Seoul National University Hospital, 101 Daehakro, Jongno-gu, Seoul 03080, Korea

Tel: +82-2-2072-2527

Fax: +82-2-765-3317

E-mail: leodavinci@naver.com

This is an Open-Access article distributed under the terms of the Creative Commons Attribution NonCommercial License (http://creativecommons.org/ licenses/by-nc/4.0) which permits unrestricted noncommercial use, distribution, and reproduction in any medium, provided the original work is properly cited.

Copyright $\odot$ 2018 The Korean Association for Radiation Protection

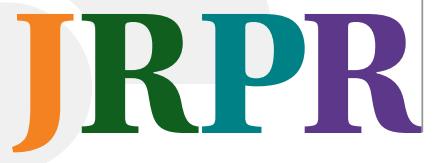

Background: We analyzed changes in the doses, structure volumes, and dose-volume histograms (DVHs) when data were transferred from one commercial treatment planning system (TPS) to another commercial TPS.

Materials and Methods: A total of 22 volumetric modulated arc therapy (VMAT) plans for nasopharyngeal cancer were generated with the Eclipse system using 6-MV photon beams. The computed tomography (CT) images, dose distributions, and structure information, including the planning target volume (PTV) and organs at risk (OARs), were transferred from the Eclipse to the MRIdian system in digital imaging and communications in medicine (DICOM) format. Thereafter, DVHs of the OARs and PTVs were generated in the MRIdian system. The structure volumes, dose distributions, and DVHs were compared between the MRIdian and Eclipse systems.

Results and Discussion: The dose differences between the two systems were negligible (average matching ratio for every voxel with a $0.1 \%$ dose difference criterion $=100.0 \pm 0.0 \%)$. However, the structure volumes significantly differed between the MRIdian and Eclipse systems (volume differences of $743.21 \pm 461.91 \%$ for the optic chiasm and $8.98 \pm 1.98 \%$ for the PTV). Compared to the Eclipse system, the MRIdian system generally overestimated the structure volumes (all, $p<0.001$ ). The DVHs that were plotted using the relative structure volumes exhibited small differences between the MRIdian and Eclipse systems. In contrast, the DVHs that were plotted using the absolute structure volumes showed large differences between the two TPSs.

Conclusion: DVH interpretation between two TPSs should be performed using DVHs plotted with the absolute dose and absolute volume, rather than the relative values.

Keywords: Dose-volumetric parameter, Treatment planning system, Volumetric-modulated arc therapy, Calculation algorithms

\section{Introduction}

Intensity-modulated radiation therapy (IMRT) and volumetric modulated arc therapy (VMAT) can generate highly conformal dose distributions through inverse planning algorithms and photon beam modulations [1, 2]. These advanced radiotherapy techniques have increased the complexity of treatment plans by improving the dose conformity for the planning target volume (PTV) while simultaneously reducing doses to surrounding normal tissue. ${ }^{1)}$

1) Kulkarni BS, et al. A prospective study of OAR volume variations between two different treatment planning systems in radiotherapy. Int. J. Radiat. Oncol. Biol. Phys. 2015;3(3). 
When a radiotherapy institution uses multiple treatment planning systems (TPS), dose distributions and structures generated from each TPS should be compared since each commercial TPS use its own algorithm to reconstruct dose distributions and structures. This can result in different outcomes for the doses and structures despite the identical input. Moreover, potential differences in the algorithms of different TPSs should be considered when a patient is transferred from one radiotherapy institution to another institution. In this case, the current institution should review the patient's treatment history to assess the doses delivered to the patient. To review the patient's treatment history, computed tomography (CT) images, dose distributions, and structures generated by the TPS of the former institution should be imported to the TPS of the current institution. The dose differences owing to different algorithms used by the TPSs are likely more significant for IMRT or VMAT plans than for 3D conformal radiation therapy (3D CRT) plans as the dose distributions of IMRT or VMAT are generally more complicated than those of the 3D CRT. Differences in the dose distributions and structures for an identical patient with an identical plan among various TPSs might result in the misinterpretation of the treatment plans as these differences affect the dose-volume histograms (DVH), which are frequently used to evaluate treatment plans [3]. If the clinically relevant dose volumetric parameters of the OARs (or PTVs) are close to the tolerance levels, this can be problematic in the clinic [4]. In this regard, several groups have studied the differences in DVHs generated by various commercial TPSs $[5,6] .{ }^{1)}$

Ebert et al. [5] investigated variations in DVH data provided by multiple TPSs and reported that the total volume and DVH data calculations were consistent among TPSs but showed small discrepancies that tended to increase with a decreasing size of the structure. Ackelry et al. [6] reported discrepancies for structure volume calculations (6-12\%) among 4 commercially available TPSs. Kulkarni et al. reported differences in the absolute volumes of the OARs (0.25$319.73 \mathrm{~mL}$ ) between the Eclipse ${ }^{\mathrm{TM}}$ and $\mathrm{XIO}^{\mathrm{TM}} \mathrm{CMS}$ systems. ${ }^{1)}$ These studies suggest that comparative analyses of different TPSs are needed to examine systematic variations in DVH data [5].

The ViewRay ${ }^{\mathrm{TM}}$ system (ViewRay Inc., Cleveland, OH), provides a unique combination of a magnetic resonance imaging system and a tri-Co-60 radiation therapy system [7]. The TPS of the ViewRay system is the MRIdian ${ }^{\mathrm{TM}}$ (version
4.1.1; ViewRay Inc., Cleveland, OH, USA); its dose calculation algorithm is based on the Monte Carlo simulation [7-10]. Based on previous studies that showed differences in DVHs among commercially available TPSs, the MRIdian system should also be evaluated. Therefore, this study evaluated the discrepancies in doses, structure volumes, and DVHs between the Eclipse and MRIdian systems when planning datasets were transferred from the former to the latter. As the Eclipse system has been already validated for DVH differences $[6],{ }^{1)}$ we used the results of the Eclipse system as a reference.

\section{Materials and Methods}

\section{Patient data and planning}

This study evaluated data from 22 patients with nasopharyngeal cancer who were treated with VMAT. Each patient underwent CT scans at a slice thickness of $3 \mathrm{~mm}$ using the Brilliance CT Big Bore ${ }^{\mathrm{TM}}$ system (Phillips, Cleveland, OH). To generate VMAT plans, structures including the PTVs and OARs (both lenses of the eyes, both optic nerves, the optic chiasm, brainstem, spinal cord, and both parotid glands) were contoured in the Eclipse system. For each patient, VMAT plans were generated with the Eclipse system, which uses 6-MV photon beams and the Millennium $120^{\mathrm{TM}}$ MLC (Varian Medical Systems, Palo Alto, CA). The prescription doses of $67.5 \mathrm{~Gy}$ (daily dose = 2.25 Gy), $54 \mathrm{~Gy}$ (daily dose $=1.8$ Gy), and 48 Gy (daily dose=1.6 Gy) were delivered to the $\mathrm{PTV}_{67.5 \mathrm{G} y}, \mathrm{PTV}_{54 \mathrm{~Gy}}$, and PTV $\mathrm{PTBG}_{4 \mathrm{y}}$ with the simultaneous integrated boost technique. Optimization of each VMAT plan was based on the normal tissue tolerance according to the Radiation Therapy Oncology Group 0615 protocol, using the progressive resolution optimizer 3 algorithm (version 10.0; Varian Medical Systems, Palo Alto, CA) [11]. After optimization, the dose distributions were calculated using the anisotropic analytic algorithm (version 10.0; Varian Medical Systems, Palo Alto, CA), with a dose calculation grid size of 1 $\mathrm{mm}$. Every VMAT plan in this study was normalized to cover $95 \%$ of the PTV $_{67.5 G y}$ volume with $95 \%$ of the prescribed dose of $67.5 \mathrm{~Gy}$. After calculating the dose distributions, the DVHs of each structure were calculated.

\section{Data transfer from the Eclipse to the MRIdian system}

The Eclipse system uses digital imaging and communications in medicine (DICOM) protocols for data transfer. ${ }^{2)}$ Thus, the patients' CT images, the structure sets contoured 
in the Eclipse system, and the dose distributions calculated in the Eclipse system were exported as DICOM files. Using the International Electrotechnical Commission coordinate system, the voxel size of the CT images in the Eclipse system was $1.367 \mathrm{~mm}(x) \times 3.000 \mathrm{~mm}(y) \times 1.367 \mathrm{~mm}(z)$, and the dose grid spacing for the Eclipse system was $1.000 \mathrm{~mm}$ $(x) \times 1.000 \mathrm{~mm}(y) \times 1.000 \mathrm{~mm}(z)$. The DICOM-formatted files but no plan information were imported into the MRIdian system. The MRIdian system has a DICOM import application to manage images, structures, and doses. When the MRIdian system imports DICOM RT data, it uses the equipment's DICOM conformance statements to perform the first step of evaluating interconnectivity and interoperability between the MRIdianand other DICOM-compliant equipment. Connectivity between the two pieces of equipment can be assessed in advance using the equipment's DICOM conformance statements. ${ }^{3)}$ The voxel size of the CT images in the MRIdian system was identical to that in the Eclipse system. The dose grid spacing for the MRIdian system was $1.367 \mathrm{~mm}$ $(x) \times 3.000 \mathrm{~mm}(y) \times 1.367 \mathrm{~mm}(z)$.

\section{Evaluation of doses, volumes, and dose-volume histograms}

After the DICOM data transfer, the DVHs were generated in the MRIdian system based on the imported dose distributions and structures but using the system's own DVH generation algorithm. For the OARs (lenses, optic nerves, optic chiasm, brainstem, spinal cord, and parotid glands) and the PTV $_{67.5 G y}$, the structure volumes and DVHs generated by the Eclipse system were compared to those generated by the MRIdian system.

We categorized the structures according to their volumes (structures with volumes $<1 \mathrm{~mL}$ vs. structures with volumes $\geq 1 \mathrm{~mL}$ ). Mean, maximum, and minimum doses were analyzed as dose-volumetric parameters for the $\mathrm{PTV}_{67.5 \mathrm{~Gy}}$. For each lens, each optic nerve, the brainstem, and the spinal cord, the maximum doses were analyzed, while the mean doses were analyzed for each parotid gland.

The RT dose files from the Eclipse system were reconstructed (not calculated) in the MRIdian system. The dose distributions of the two systems were compared with the 3DVHTM software (Sun Nuclear Corporation, Melbourne, FL) [12], which can compare entire 3D dose distributions from multiple TPSs. RT dose files were exported from the
Eclipse and MRIdian systems and imported into the 3DVH software. The dose distributions generated by the Eclipse system were used as the reference, whereas the dose distribution generated by the MRIdian system were used as the comparison. The 3D dose comparison was executed voxel by voxel, with a dose difference criterion of $0.1 \%$ of the prescription dose. When 3DVH compared the dose distributions with different grid size, the spatial resolution of that by MRIdian was resampled to the reference data. A paired $t$ test was used to assess statistically significant differences in the results from the two TPSs.

\section{Results and Discussion}

\section{Dose differences}

The average matching ratio for each voxel was $100.0 \% \pm$ $0.0 \%$, and the average dose difference ranged from $-2.98 \mathrm{cGy}$ to $4.03 \mathrm{cGy}$ for every patient in this study. The median absolute dose difference was $0.2 \mathrm{cGy}$. The dose differences between the two TPSs were considered negligible.

\section{Volume differences}

The volumes of the analyzed structures in this study ranged from $0.01 \mathrm{~mL}$ (optic chiasm) to $289.7 \mathrm{~mL}$ (PTV $67.5 \mathrm{~Gy}$ ). For every structure in this study, the volumes calculated by the Eclipse system were significantly different from those calculated by the MRIdian system (Table 1). The structure volumes calculated by the MRIdian system were significantly larger than those calculated by the Eclipse system (all, $p<0.001)$.

\section{Dose-volume histogram differences}

Table 2 shows the average dosimetric parameters of the Eclipse and MRIdian systems as well as the relative differences between the two systems. We observed statistically significant differences in the $\mathrm{PTV}_{67.5 \mathrm{~Gy}}$ and both parotid glands between the Eclipse and MRIdian systems (all, $p<0.001$ ). The dose-volumetric parameters of the PTV $\mathrm{P}_{67.5 \mathrm{~Gy}}$ calculated by the Eclipse system were higher than those calculated by the MRIdian system ( $4.84 \%$ difference with $p<0.001$ for the minimum dose, $0.37 \%$ difference with $p<0.001$ for the maximum dose, and $0.21 \%$ difference with $p<0.001$ for the mean dose). In contrast, the dose-volumetric parameters of both parotid glands calculated by the MRIdian system were high-

2) Eclipse Treatment Planning System Feature Sheet.

3) Varian Medical Systems 2011 System Server-DICOM Conformance Statement (VA10003D3CS). 
Table 1. Average Structure Volumes and Relative Differences

\begin{tabular}{|c|c|c|c|c|}
\hline & Eclipse $^{\mathrm{TM}}$ system (mL) & MRIdian ${ }^{\mathrm{TM}}$ system (mL) & Relative difference (\%) & $p$ \\
\hline \multicolumn{5}{|c|}{ Structure volumes $<1 \mathrm{~mL}$} \\
\hline Right lens & $0.18 \pm 0.09$ & $0.35 \pm 0.13$ & $107.52 \pm 59.34$ & $<0.001$ \\
\hline Left lens & $0.16 \pm 0.07$ & $0.35 \pm 0.11$ & $133.26 \pm 69.39$ & $<0.001$ \\
\hline Right optic nerve & $0.28 \pm 0.14$ & $0.75 \pm 0.25$ & $211.39 \pm 125.14$ & $<0.001$ \\
\hline Left optic nerve & $0.31 \pm 0.15$ & $0.79 \pm 0.25$ & $186.25 \pm 83.80$ & $<0.001$ \\
\hline Optic chiasm & $0.15 \pm 0.17$ & $0.75 \pm 0.35$ & $743.21 \pm 461.91$ & $<0.001$ \\
\hline \multicolumn{5}{|c|}{ Structure volumes $\geq 1 \mathrm{~mL}$} \\
\hline PTV67.5Gy & $147.30 \pm 111.86$ & $159.43 \pm 120.51$ & $8.98 \pm 1.98$ & $<0.001$ \\
\hline Brainstem & $27.32 \pm 5.57$ & $30.88 \pm 6.03$ & $13.22 \pm 1.32$ & $<0.001$ \\
\hline Right parotid gland & $22.26 \pm 7.88$ & $25.38 \pm 8.51$ & $14.97 \pm 3.04$ & $<0.001$ \\
\hline Left parotid gland & $21.63 \pm 7.71$ & $24.67 \pm 8.31$ & $14.95 \pm 2.82$ & $<0.001$ \\
\hline Spinal cord & $20.78 \pm 4.74$ & $25.54 \pm 5.88$ & $23.46 \pm 11.06$ & $<0.001$ \\
\hline
\end{tabular}

PTV67.5Gy, planning target volume with a prescription dose of $67.5 \mathrm{~Gy}$.

Table 2. Average Dose-volumetric Parameters and Relative Differences

\begin{tabular}{lrcrrr}
\hline & Parameter & Eclipse $^{\mathrm{TM}}$ system & MRldian ${ }^{\mathrm{TM}}$ systme & Relative difference (\%) & \multicolumn{1}{c}{$p$} \\
\hline PTV67.5Gy & Min (Gy) & $59.56 \pm 5.06$ & $56.83 \pm 6.63$ & $-4.84 \pm 3.90$ & $<0.001$ \\
& Max (Gy) & $73.38 \pm 1.78$ & $73.10 \pm 1.70$ & $-0.37 \pm 0.39$ & $<0.001$ \\
Brainstem & Mean (Gy) & $69.30 \pm 0.59$ & $69.16 \pm 0.57$ & $-0.21 \pm 0.06$ & $<0.001$ \\
Spinal cord & Max (Gy) & $52.26 \pm 2.35$ & $52.48 \pm 2.56$ & $0.41 \pm 1.33$ & 0.162 \\
Right lens & Max (Gy) & $42.05 \pm 2.09$ & $42.22 \pm 2.08$ & $0.41 \pm 1.08$ & 0.101 \\
Left lens & Max (Gy) & $4.80 \pm 0.87$ & $4.80 \pm 0.87$ & $0.01 \pm 0.70$ & 0.849 \\
Right optic nerve & Max (Gy) & $4.87 \pm 0.94$ & $4.86 \pm 0.92$ & $-0.06 \pm 1.15$ & 0.599 \\
Left optic nerve & Max (Gy) & $22.13 \pm 14.09$ & $22.11 \pm 14.10$ & $-0.13 \pm 1.92$ & 0.764 \\
Optic chiasm & Max (Gy) & $23.74 \pm 14.48$ & $23.73 \pm 14.54$ & $-0.22 \pm 1.88$ & 0.831 \\
Right parotid gland & Max (Gy) & $27.14 \pm 14.89$ & $26.87 \pm 14.93$ & $-1.26 \pm 2.74$ & 0.054 \\
Left parotid gland & Mean (Gy) & $24.63 \pm 1.03$ & $25.22 \pm 1.00$ & $2.44 \pm 1.12$ & $<0.001$ \\
\hline
\end{tabular}

Min, minimum dose; Max, maximum dose; Mean, mean dose; PTV67.5Gy, planning target volume with a prescription dose of 67.5 Gy.

er than those calculated by the Eclipse system ( $2.44 \%$ difference with $p<0.001$ for the mean dose of the right parotid gland and $2.89 \%$ difference with $p<0.001$ for the mean dose of the left parotid gland). However, the maximum dose differences for the other OARs were $<1 \%$, regardless of the volumes (all, $p>0.05$ ).

Figure 1 shows a representative comparison of DVHs that were plotted using the absolute doses and relative volumes of the structures. The differences in the DVHs were more significant for structures with volumes $<1 \mathrm{~mL}$ (Figure 1B). Figure 2 shows the differences in the same DVHs, but plotted using the absolute doses and absolute volumes. Based on the significant absolute volume differences of the structures between the Eclipse and MRIdian systems, noticeable differences in the DVHs between the two systems were observed. The differences in the DVHs using the absolute volumes were more significant for structures with volumes $<1 \mathrm{~mL}$
(Figure 2B).

This study evaluated the discrepancies in doses, volumes, and DVHs between the Eclipse and MRIdian systems. We found significant differences in the structure volumes but not in the doses between the two TPSs. The differences in the structure volumes resulted in differences in the DVHs. For the DVHs that were plotted using the relative volumes, the $\mathrm{PTV}_{67.5 \mathrm{~Gy}}$ and both parotid glands showed statistically significant differences between the Eclipse and MRIdian systems. For the DVHs that were plotted with the absolute structure volumes, we also observed large differences between the Eclipse and MRIdian systems, particularly for small structures such as the lens, optic nerves, and optic chiasm.

Commercially available TPSs calculate a 3D structure volume by interpolating 2D contours defined at the planar images (e.g., CT or magnetic resonance imaging), using their 

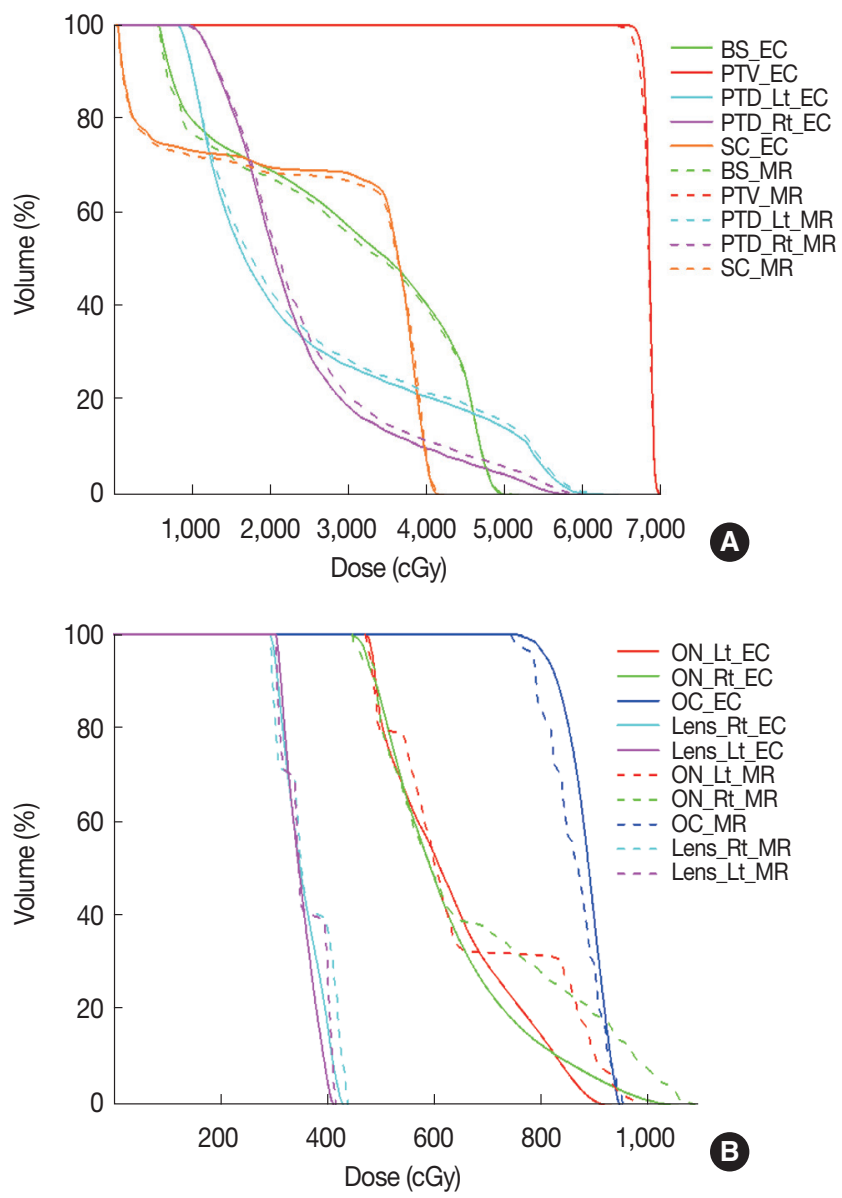

Fig. 1. Representative dose-volume histogram comparison, plotted using the absolute dose and relative volume. This dose-volume histogram (DVH) comparison revealed a small difference in each relative volume that received the identical dose. The difference was more significant for structure volumes $<1 \mathrm{~mL}(\mathrm{~B})$, when compared to structure volumes $\geq 1 \mathrm{~mL}$ (A). BS, brainstem; EC, Eclipse; Lt, left; MR, MRIdian; OC, optic chiasm; ON, optic nerve; PTD, parotid gland; PTV, planning target volume; Rt, right; SC, spinal cord.

own interpolation algorithms. For example, the Eclipse system uses the shape-based interpolation algorithm to calculate structure volumes, based on the correspondence to an object's shape and position in the adjacent slices [13, 14]. On the contrary, the MRIdian system uses the linear-interpolation algorithm to calculate 3D structure volumes. Differences in the algorithms that are used to define the 3D structures between the Eclipse and MRIdian systems result in different volumes of identical structure. This seems to mainly contribute to different DVH values for identical structures that are calculated by the two systems, as the DVH values are affected by the structure volumes. Differences in the dose distributions between the Eclipse and MRIdian systems might also
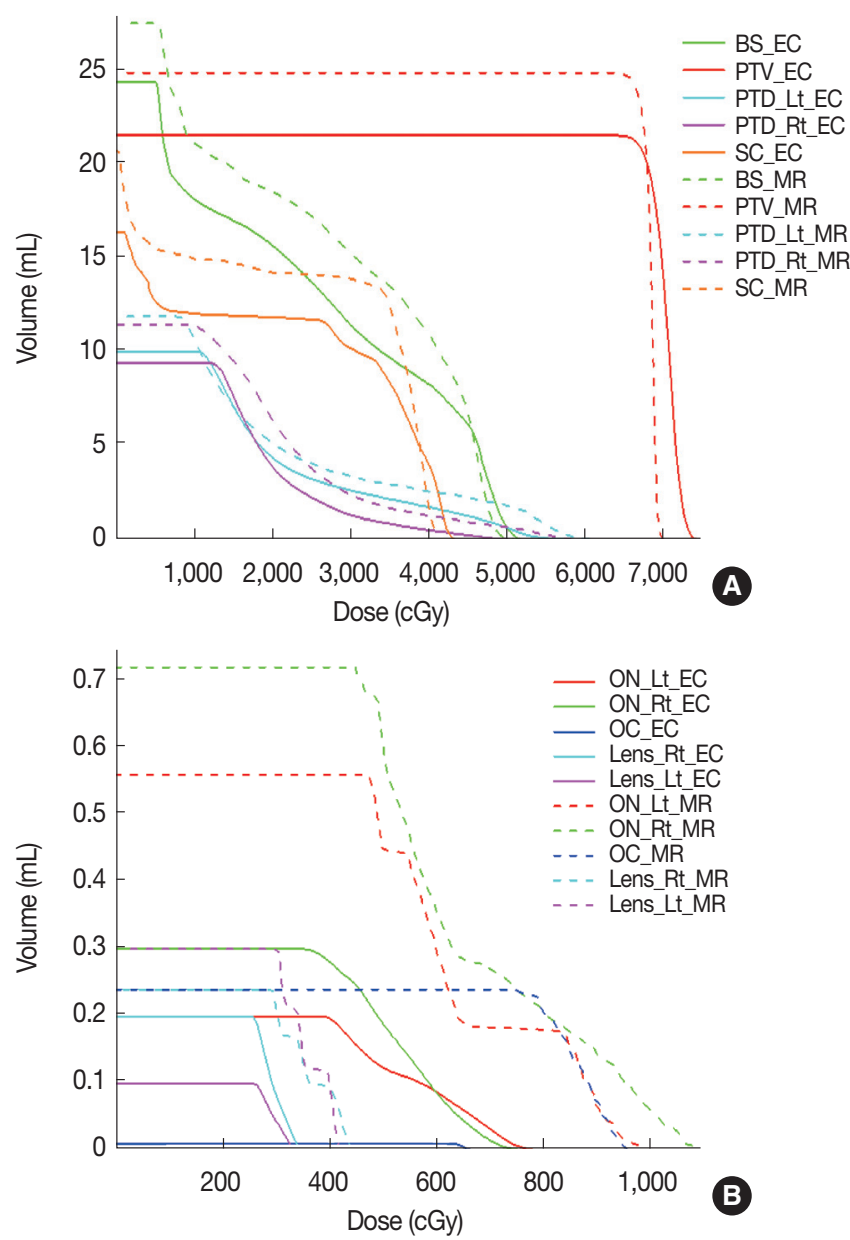

Fig. 2. Representative dose-volume histogram comparison, plotted using the absolute dose and absolute volume. Due to significant differences in the absolute volumes of the structures, this dose-volume histogram $(\mathrm{DVH})$ comparison revealed a remarkable difference for any volume that received the identical dose. This difference was more significant for structure volumes $<1 \mathrm{~mL}(\mathrm{~B})$, when compared to structure volumes $\geq 1 \mathrm{~mL}(\mathrm{~A})$. BS, brain stem; EC, Eclipse; Lt, left; MR, MRIdian; OC, optic chiasm; ON, optic nerve; PTD, parotid gland; PTV, planning target volume; Rt, right; SC, spinal cord.

cause different DVH values; however, our study showed that the dose distributions of the Eclipse system were almost identical to those of the MRIdian system. Therefore, different DVH values between the Eclipse and MRIdian system are likely caused by differences in the structure volumes. It is also possible that differences in the DVH calculation algorithms between the two systems might have caused differences in the DVH values.

Although we observed some discrepancies in the DVHs that were plotted using the relative volumes between the two TPSs, since the MRIdian system is a commercially available TPS, we observed no clinically significant differences in the DVHs between the Eclipse and MRIdian systems. We detect- 
ed no clinically relevant but statistically significant differences in the DVHs of the PTV 67.5 Gy and of both parotid glands, for which the dose fall-offs were generally large in the head and neck VMAT plans with simultaneous integrated boost techniques. Therefore, when evaluating the DVHs of structures that are located at the steep dose fall-off in the MRIdian system, it seems advisable to consider some uncertainty regarding the dose-volumetric parameters.

For the DVHs that were plotted using the absolute volumes and absolute doses, the differences in the DVHs between the MRIdian and Eclipse system were large due to the large volume differences. For fractionated radiotherapy, normal tissue tolerances based on literature or guidelines are provided with relative volumes of the OARs that are irradiated by certain doses $[15,16]$. However, for stereotactic ablative radiotherapy (SABR), normal tissue tolerances are generally provided with the absolute volumes of the OARs that are irradiated by certain doses [17-19]; therefore, when performing SABR with the MRIdian system, it seems advisable to be cautious when evaluating the dose-volumetric parameters, particularly for small structures, as they showed large differences in the DVHs that were plotted using absolute volumes between the Eclipse and MRIdian systems in this study. Furthermore, if only one TPS is available in your clinic, you need to establish your own data about the accuracy of the volume definition.

\section{Conclusion}

The present study demonstrated that the MRIdian system tended to overestimate the structure volumes, when compared to the Eclipse system. Despite the differences in the dose calculation resolutions between the two systems, no significant dose differences were observed. Due to the differences in the structure volumes, the DVHs that were plotted using the absolute volumes showed large differences between the Eclipse and MRIdian systems, particularly for small structures.

\section{Acknowledgements}

This work was supported by National Research Foundation of Korea grants, which are funded by the Korea government (MSIP; 2017M2A2A7A02020641 and 2017M2A2A7A 02020643).

\section{References}

1. Gintz D, Latifi K, Caudell J, Nelms B, Zhang G, Moros E, Feygelman V. Initial evaluation of automated treatment planning software. J. Appl. Clin. Med. Phys. 2016;17(3):331-346.

2. Ostheimer C, Hubsch P, Janich M, Gerlach R, Vordermark D. Dosimetric comparison of intensity-modulated radiotherapy (IMRT) and volumetric modulated arc therapy (VMAT) in total scalp irradiation: a single institutional experience. Radiat. Oncol. J. 2016;34(4):313-321.

3. Alfonso JC, Herrero MA, Nunez L. A dose-volume histogram based decision-support system for dosimetric comparison of radiotherapy treatment plans. Radiat. Oncol. 2015;10:263.

4. Drzymala RE, Mohan R, Brewster L, Chu J, Goitein M, Harms W, Urie M. Dose-volume histograms. Int. J. Radiat. Oncol. Biol. Phys. 1991;21(1):71-78

5. Ebert MA, Haworth A, Kearvell R, Hooton B, Hug B, Spry NA, Bydder SA, Joseph DJ. Comparison of DVH data from multiple radiotherapy treatment planning systems. Phys. Med. Biol. 2010; 55(11):N337-346.

6. Ackerly T, Andrews J, Ball D, Guerrieri M, Healy B, Williams I. Discrepancies in volume calculations between different radiotherapy treatment planning systems. Australas. Phys. Eng. Sci. Med. 2003;26(2):91-93.

7. Dempsey JF, et al. A device for realtime 3D image-guided IMRT. Int. J. Radiat. Oncol. Biol. Phys. 2005;63(2):S202.

8. Bostel T, Nicolay NH, Grossmann JG, Mohr A, Delorme S, Echner G, Häring P, Debus J, Sterzing F. MR-guidance - a clinical study to evaluate a shuttle- based MR-linac connection to provide MRguided radiotherapy. Radiat. Oncol. 2014;9:12-19.

9. Yang D, Wooten HO, Green O, Li HH, Liu S, Li x, Rodriguez V, Mutic S, Kashani R. A software tool to automatically assure and report daily treatment deliveries by a cobalt-60 radiation therapy device. J. Appl. Clin. Med. Phys. 2016;17(3):492-501.

10. Mutic S, Dempsey JF. The ViewRay system: magnetic resonanceguided and controlled radiotherapy. Semin. Radiat. Oncol. 2014; 24(3):196-199.

11. Muralidhar KR, Pangam S, Srinivas P, Ali MA, Priya VS, Komanduri K. A phantom study on the behavior of Acuros XB algorithm in flattening filter free photon beams. J. Med. Phys. 2015;40(3): 144-149.

12. Olch AJ. Evaluation of the accuracy of 3DVH software estimates of dose to virtual ion chamber and film in composite IMRT QA. Med. Phys. 2012;39(1):81-86.

13. Lee TY, Lin CH. Feature-guided shape-based image interpolation. IEEE Trans. Med. Imaging. 2002;21(12):1479-1489.

14. Chuang KS, Chen CY, Yuan LJ, Yeh CK. Shape-based grey-level image interpolation. Phys. Med. Biol. 1999;44(6):1565-1577.

15. Emami B, Lyman J, Brown A, Cola L, Goitein M, Munzenrider 
JE, Shank B, Solin LJ, Wesson M. Tolerance of normal tissue to therapeutic irradiation. Int. J. Radiat. Oncol. Biol. Phys. 1991; 21(1):109-122.

16. Marks LB, Yorke ED, Jackson A, Ten Haken RK, Constine LS, Eisbruch A, Bentzen SM, Nam J, Deasy JO. Use of normal tissue complication probability models in the clinic. Int. J. Radiat. Oncol. Biol. Phys. 2010;76(3):S10-S19.

17. Huang Z, Mayr NA, Yuh WT, Wang JZ, Lo SS. Reirradiation with stereotactic body radiotherapy: Analysis of human spinal cord tolerance using the generalized linear-quadratic model. Future Oncol. 2013;9(6):879-887.

18. Sahgal A, et al. Reirradiation human spinal cord tolerance for stereotactic body radiotherapy. Int. J. Radiat. Oncol. Biol. Phys. 2012;82(1):107-116.

19. Timmerman RD. An overview of hypofractionation and introduction to this issue of seminars in radiation oncology. Seminars in Radiation Oncology. 2008;18(4):215-222. 